\title{
Brill-Noether theory on singular curves and torsion-free sheaves on surfaces
}

\author{
TOMÁs L. Gómez
}

Let $C$ be a smooth curve of genus $g$. Let $W_{d}^{r}(C)$ be the BrillNoether locus of line bundles of degree $d$ and with $r+1$ independent sections. The expected dimension of $W_{d}^{r}(C)$ is $\rho(r, d)=g-(r+$ 1) $(g-d+r)$. If $\rho(r, d)>0$ then Fulton and Lazarsfeld have proved that $W_{d}^{r}(C)$ is connected. We prove that this is still true if $C$ is a singular irreducible curve lying on a regular surface $S$ with $-K_{S}$ generated by global sections.

We use this result to give a short new proof of the irreducibility of the moduli space of rank 2 semistable torsion-free sheaves (with a generic polarization and low value of $c_{2}$ ) on a K3 surface (this result was recently proved by a different method by O'Grady).

\section{Introduction.}

Let $C$ be a smooth curve of genus $g$ (we will always assume that the base field is $\mathbb{C}), J(C)$ its Jacobian, and $W_{d}^{r}(C)$ the Brill-Noether locus corresponding to line bundles $L$ of degree $d$ and $h^{0}(L) \geq r+1$ (see [ACGH]). The expected dimension of this subvariety is $\rho(r, d)=g-(r+1)(g-d+r)$. Fulton and Lazarsfeld [F-L] proved that $W_{d}^{r}(C)$ is connected when $\rho>0$. We are going to generalize this result for certain singular curves, but before stating our result (Theorem I), we need to recall some concepts.

Let $C$ be an integral curve (not necessarily smooth). We still have a generalized Jacobian $J(C)$, defined as the variety parametrizing line bundles, but it will not be complete in general. Define the degree of a rank one torsion-free sheaf on $C$ to be

$$
\operatorname{deg}(A)=\chi(A)+p_{a}-1,
$$

where $p_{a}$ is the arithmetic genus of $C$. One can define a scheme $\bar{J}^{d}(C)$ parametrizing rank one torsion-free sheaves on $C$ of degree $d$ (see [AIK], [D], [R]). If $C$ lies on a surface, then $\bar{J}^{d}(C)$ is integral, and furthermore the generalized Jacobian $J(C)$ is an open set in $\bar{J}^{d}(C)$, and then $\bar{J}^{d}(C)$ is a natural compactification of $J(C)$. 
We can define the generalized Brill-Noether locus $\bar{W}_{d}^{r}(C)$ as the set of points in $\bar{J}^{d}(C)$ corresponding to sheaves $A$ with $h^{0}(A) \geq r+1$ (note that it is complete because of the upper semicontinuity of $\left.h^{0}(\cdot)\right)$. There is also a determinantal description that gives a scheme structure. This description is a straightforward generalization of the description for smooth curves (see [ACGH]), but we are only interested in the connectivity of $\bar{W}_{d}^{r}(C)$, so we can give it the reduced scheme structure.

We will consider curves that lie on a surface $S$ with the following property:

$$
h^{1}\left(\mathcal{O}_{S}\right)=0, \text { and }-K_{S} \text { is generated by global sections. }
$$

We will need this condition to prove Proposition 2.5. For instance, $S$ can be a K3 surface. Now we can state the theorem that we are going to prove.

Theorem I. Let $C$ be a reduced irreducible curve of arithmetic genus $p_{a}$ that lies in a surface $S$ satisfying $(*)$. Let $\bar{J}^{d}(C), d>0$, be the compactification of the generalized Jacobian. Then for any $r \geq 0$ such that $\rho(r, d)=p_{a}-$ $(r+1)\left(p_{a}-d+r\right)>0$, the generalized Brill-Noether subvariety $\bar{W}_{d}^{r}(C)$ is nonempty and connected.

Remark 1. If $r \leq d-p_{a}$, by Riemann-Roch inequality we have $\bar{W}_{d}^{r}(C)=$ $\bar{J}^{d}(C)$, and this is connected. Then, in order to prove theorem I we can assume $r>d-p_{a}$. Note that if $A$ corresponds to a point in $\bar{W}_{d}^{r}(C)$ with $r>d-p_{a}$, then by Riemann-Roch theorem $h^{1}(A)>0$.

Let $S$ be a K3 complex surface and $H$ an ample line bundle. Let $\mathfrak{M}_{H}\left(c_{1}, c_{2}\right)$ be the moduli space of rank two torsion-free sheaves that are Gieseker semistable with respect to $H$, with Chern classes equal to $c_{1}$ and $c_{2}$. As an application of theorem I we give a new short proof of the following.

Theorem II. With the previous notation, if $L$ is a primitive big and nef line bundle, $c_{2} \leq \frac{1}{2} L^{2}+3$, and $H$ is an $\left(L, c_{2}\right)$-generic polarization, then $\mathfrak{M}_{H}\left(L, c_{2}\right)$ is irreducible.

For the definition of $\left(L, c_{2}\right)$-generic and for the proof of Theorem II see Section 5. Mukai [M] has proved irreducibility when the dimension is 0 or 2 . In general for any surface, it is known that for a fixed polarization and $c_{1}$, the moduli space is irreducible for high enough second Chern class $c_{2}$ ([G-L], 
[O1], and [O2]). The case of a K3 surface has also been studied by O'Grady [O3], that has proved irreducibility (for any $c_{2}$ and for any rank) as well as results about the Hodge structure, using a different method. Göttsche and Huybrechts [G-H] have studied the Hodge numbers of this moduli space. Yoshioka also has a paper [Yo] on bundles on K3 surfaces in which he proves irreducibility of the moduli space among other things.

To prove Theorem I for a curve $C$ satisfying $(*)$ we will construct a deformation of $C$ into a smooth curve and we will use the fact that FultonLazarsfeld's theorem holds for smooth curves to show that it also holds for $C$. Given a family of curves we will need to construct a corresponding family of generalized Jacobians and Brill-Noether loci. All this can be done using a relative version of $\bar{J}^{d}(C)$, but we will proceed in a different way. We will use the fact that all these curves are going to lie on a fixed surface $S$. Then we will think of the coherent sheaves on $C$ as torsion sheaves on $S$ (all sheaves in this paper will be coherent). To define precisely which sheaves we will consider we need some notation. For any sheaf $F$ on $S$, let $d(F)$ be the dimension of its support. We say that $F$ has pure dimension $n$ if for any subsheaf $E$ of $F$ we have $d(E)=d(F)=n$. Note that if the support is irreducible, then having pure dimension $n$ is equivalent to being torsion-free when considered as a sheaf on its support. The following theorem follows from [S, Theorem 1.21].

Theorem (Simpson). Let $C$ be an integral curve on a surface $S$. Let $\overline{\mathcal{J}}_{|C|}^{d}$ be the functor which associates to any scheme $T$ the set of equivalence classes of sheaves $\mathcal{A}$ on $S \times T$ with

(a) $\mathcal{A}$ is flat over $T$.

(b) The induced sheaf $A_{t}$ on each fiber $S \times\{t\}$ has pure dimension 1, and its support is an integral curve in the linear system $|C|$.

(c) If we consider $A_{t}$ as a sheaf on its support, it is torsion-free and has rank one and degree $d$.

Sheaves $\mathcal{A}$ and $\mathcal{B}$ are equivalent if there exists a line bundle $L$ on $T$ such that $\mathcal{A} \cong \mathcal{B} \otimes p_{T}^{*} L$, where $p_{T}: S \times T \rightarrow T$ is the projection on the second factor.

Then there is a coarse moduli space that we also denote by $\overline{\mathcal{J}}_{|C|}^{d}$. I.e., the points of $\overline{\mathcal{J}}_{|C|}^{d}$ correspond to isomorphism classes of sheaves, and for any family $\mathcal{A}$ of such sheaves parametrized by $T$, there is a morphism

$$
\phi: T \rightarrow \overline{\mathcal{J}}_{|C|}^{d}
$$


such that $\phi(t)$ corresponds to the isomorphism class of $A_{t}$.

Note that $\overline{\mathcal{J}}_{|C|}^{d}$ parametrizes pairs $\left(C^{\prime}, A\right)$ with $C^{\prime}$ an integral curve linearly equivalent to $C$ and $A$ a torsion-free rank one sheaf on $C$.

We denote by $\pi: \overline{\mathcal{J}}_{|C|}^{d} \rightarrow U \subset|C|$ the obvious projection giving the support of each sheaf, where $U$ is the open subset of $|C|$ corresponding to integral curves.

A family of curves on a surface $S$ parametrized by a curve $T$ is a subvariety $\mathcal{C} \subset S \times T$, flat over $T$, such that the fiber $\left.\mathcal{C}\right|_{t}=C_{t}$ over each $t \in T$ is a curve on $S$. Analogously, a family of sheaves on a surface $S$ parametrized by a curve $T$ is a sheaf $\mathcal{A}$ on $S \times T$, flat over $T$. For each $t \in T$ we will denote the corresponding member of the family by $A_{t}=\left.\mathcal{A}\right|_{t}$.

Altman, Iarrobino and Kleiman [AIK] proved the following theorem

Theorem (Altman-Iarrobino-Kleiman). With the same notation as before, $\overline{\mathcal{J}}_{|C|}^{d}$ is flat over $U$ and its geometric fibers are integral. The subset of $\overline{\mathcal{J}}_{|C|}^{d}$ corresponding to line bundles (i.e., the relative generalized Jacobian) is open and dense in $\overline{\mathcal{J}}_{|C|}^{d}$.

We also consider the family of generalized Brill-Nother loci $\overline{\mathcal{W}}_{d,|C|}^{r} \subset$ $\overline{\mathcal{J}}_{|C|}^{d}$, and the projection $q: \overline{\mathcal{W}}_{d,|C|}^{r} \rightarrow U$.

\section{Outline of the proof of Theorem I.}

Note that $\bar{W}_{d}^{r}(C)$ is the fiber of $q$ over the point $u_{0} \in|C|$ corresponding to the curve $C$. Let $U$ be the open subset of $|C|$ corresponding to integral curves, and $V$ the subset of smooth curves. Define $\left(\overline{\mathcal{W}}_{d}^{r}\right)_{V}$ to be the BrillNoether locus of sheaves with smooth support, i.e., $\left(\overline{\mathcal{W}}_{d}^{r}\right)_{V}=q^{-1}(V)$. By [F-L], the restriction $q_{V}:\left(\overline{\mathcal{W}}_{d}^{r}\right)_{V} \rightarrow V$ has connected fibers. We want to use this fact to show that $\bar{W}_{d}^{r}(C)$ is connected. Let $A$ be a rank one torsionfree sheaf on $C$ corresponding to a point in $\bar{W}_{d}^{r}(C)$, and assume that it is generated by global sections. We think of $A$ as a torsion sheaf on $S$. Then we have a short exact sequence on $S$

$$
0 \rightarrow E \stackrel{f_{0}}{\rightarrow} H^{0}(A) \otimes \mathcal{O}_{S} \rightarrow A \rightarrow 0,
$$

where the map on the right is evaluation. This sequence has already appeared in the literature (see [L], [Ye]). Our idea is to deform $f_{0}$ to a family $f_{t}$. The cokernel of $f_{t}$ will define a family of sheaves $A_{t}$ with $h^{0}\left(A_{t}\right) \geq h^{0}(A)$ (because $h^{0}(E)=0$ ), and then for each $t$ the point in $\overline{\mathcal{J}}_{|C|}^{d}$ corresponding 
to $A_{t}$ lies in $\overline{\mathcal{W}}_{d,|C|}^{r}$. Assume that there are 'enough' homomorphisms from $E$ to $H^{0} \otimes \mathcal{O}_{S}$ and the family $f_{t}$ can be chosen general enough, so that for a general $t$, the support of $A_{t}$ is smooth (the details of this construction are in Section 2). The family $A_{t}$ shows that the point in $\bar{W}_{d}^{r}(C)$ corresponding to $A$ is in the closure of $\left(\overline{\mathcal{W}}_{d}^{r}\right)_{V}$ in $\overline{\mathcal{J}}_{|C|}^{d}$. It can be shown that this closure has connected fibers. Let $X$ be the fiber over $u_{0}$ of this closure. Then all sheaves for which this construction works are in the connected component $X$ of $\bar{W}_{d}^{r}(C)$. If this could be done for all sheaves in $\bar{W}_{d}^{r}(C)$ this would finish the proof, but there are sheaves for which this construction doesn't work. For these sheaves we show in section 3 that they can be deformed (keeping the support $C$ unchanged) to a sheaf for which a refinement of this construction works. This shows that all points in $\bar{W}_{d}^{r}(C)$ are in the connected component $X$.

\section{The main lemma.}

The precise statement that we will use to prove Theorem I is the following lemma.

Lemma 1.1. Let $C$ be an integral complete curve in a surface $S$. Assume that for each rank one torsion-free sheaf $A$ on $C$ with $h^{0}(A)=r+1>0$ and $\operatorname{deg}(A)=d>0$ such that $\rho(r, d)>0$ we have the following data:

(a) A family of curves $\mathcal{C}$ in $S$ parametrized by an irreducible curve $T$ (not necessarily complete).

(b) A connected curve $T^{\prime}$ (not necessarily irreducible nor complete) with a $\operatorname{map} \psi: T^{\prime} \rightarrow T$.

(c) $A$ rank one torsion-free sheaf $\mathcal{A}$ on $\mathcal{C}^{\prime}=\mathcal{C} \times_{T} T^{\prime}$, flat over $T^{\prime}$, inducing rank one torsion-free sheaves on the fibers of $\mathcal{C}^{\prime} \rightarrow T^{\prime}$.

Assume that the following is satisfied:

(i) $\left.\mathcal{C}\right|_{t_{0}} \cong C$ for some $t_{0} \in T,\left.\mathcal{C}\right|_{t}$ is linearly equivalent to $C$ for all $t \in T$, and $\left.\mathcal{C}\right|_{t}$ is smooth for $t \neq t_{0}$.

(ii) One irreducible component of $T^{\prime}$ is a finite cover of $T$, and the rest of the components of $T^{\prime}$ are mapped to $t_{0} \in T$.

(iii) $\left.\mathcal{A}\right|_{t_{0}^{\prime}} \cong A$ for some $t_{0}^{\prime} \in T^{\prime}$ mapping to $t_{0} \in T$. 
(iv) $h^{0}\left(\left.\mathcal{A}\right|_{t^{\prime}}\right) \geq r+1$ for all $t^{\prime} \in T^{\prime}$.

Then the generalized Brill-Noether subvariety $\bar{W}_{d}^{r}(C)$ of the compactified generalized Jacobian $\bar{J}^{d}(C)$ is connected.

Proof. We will use the notation introduced in the previous section. The map $q: \overline{\mathcal{W}}_{d,|C|}^{r} \rightarrow U$ is a projective morphism. Recall that $\bar{W}_{d}^{r}(C)$ is the fiber of $q$ over $u_{0}$, where $u_{0}$ is the point corresponding to $C$. By [F-L] the morphism $q$ has connected fibers over $V$, thus a general fiber of $q$ is connected, and we want to prove that the fiber over $u_{0} \in U$ is also connected.

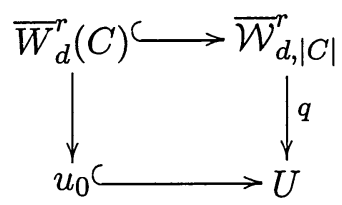

Let $\overline{\mathcal{W}}_{d,|C|}^{r} \stackrel{q^{\prime}}{\rightarrow} U^{\prime} \stackrel{g}{\rightarrow} U$ be the Stein factorization of $q$ (see [H, III Corollary 11.5]), i.e., $q^{\prime}$ has connected fibers and $g$ is a finite morphism. A general fiber of $q$ is connected, and then $U^{\prime}$ has one irreducible component $Z$ that maps to $U$ birationally. The subset $U$ is open in $|C|$ and hence normal, the restriction $\left.g\right|_{Z}: Z \rightarrow U$ is finite and birational, $Z$ and $U$ are integral, thus by Zariski's main theorem (see [H, III Corollary 11.4]) each fiber of $\left.g\right|_{Z}$ consists of just one point. Let $z_{0}$ be the point of $Z$ in the fiber $g^{-1}\left(u_{0}\right)$.

Claim. Let $y_{0}$ be a point in the fiber $q^{-1}\left(u_{0}\right)=\bar{W}_{d}^{r}(C)$. Then $y_{0}$ is mapped by $q^{\prime}$ to $z_{0}$.

This claim implies that that $\bar{W}_{d}^{r}(C)$ is connected. Now we will prove the claim.

Let $A$ be the sheaf on $S$ corresponding to the point $y_{0}$. Let $T^{\prime}, T$, $t_{0}^{\prime} \in T^{\prime}, t_{0} \in T, \psi: T^{\prime} \rightarrow T$ be the curves points and morphism given by the hypothesis of the lemma. Let $\phi: T^{\prime} \rightarrow \overline{\mathcal{J}}_{|C|}^{d}$ be the morphism given by the universal property of the moduli space $\overline{\mathcal{J}}_{|C|}^{d}$. Item (iv) imply that the 
image of $\phi$ is in $\overline{\mathcal{W}}_{d,|C|}^{r}$.
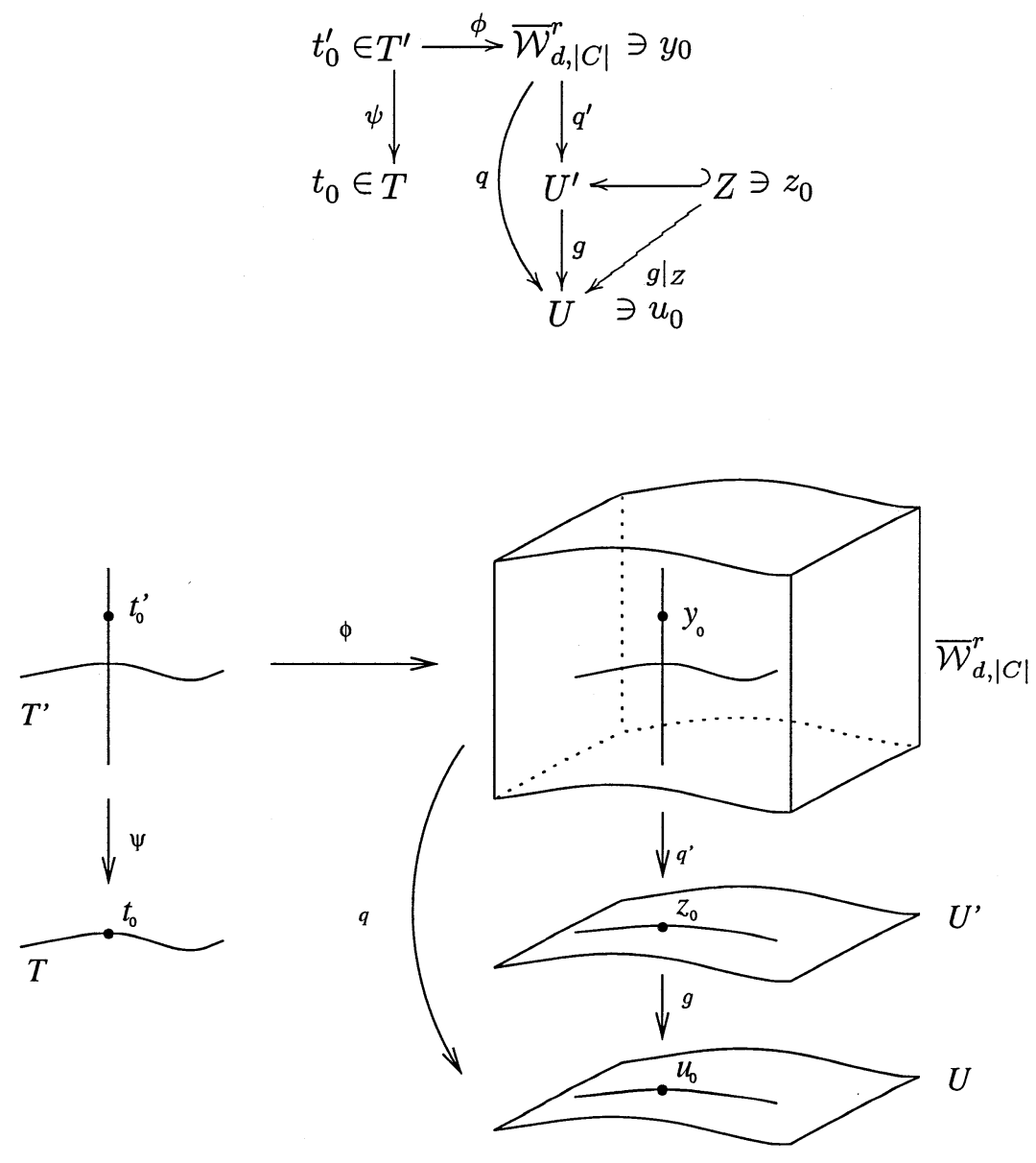

The restriction of $q^{\prime} \circ \phi$ to $T^{\prime} \backslash \psi^{-1}\left(t_{0}\right)$ maps to $Z$, because for $t^{\prime} \in$ $T^{\prime} \backslash \psi^{-1}\left(t_{0}\right)$ the sheaf $\left.\mathcal{A}\right|_{t^{\prime}}$ has smooth support by item (i). Items (c) and (i) imply that $g \circ q^{\prime} \circ \phi\left(\psi^{-1}\left(t_{0}\right)\right)=u_{0}$. Thus $q^{\prime} \circ \phi\left(\psi^{-1}\left(t_{0}\right)\right)$ is a finite number of points (because it is in the fiber of $g$ over $u_{0}$ ).

The facts that $q^{\prime} \circ \phi\left(T^{\prime} \backslash \psi^{-1}\left(t_{0}\right)\right)$ is in $Z$ and that $q^{\prime} \circ \phi\left(\psi^{-1}\left(t_{0}\right)\right)$ is a finite number of points imply that $q^{\prime} \circ \phi\left(\psi^{-1}\left(t_{0}\right)\right)$ is also in $Z$ (because by item (b) the curve $T^{\prime}$ is connected and thus also its image under $\left.q^{\prime} \circ \phi\right)$, and in fact $q^{\prime} \circ \phi\left(\psi^{-1}\left(t_{0}\right)\right)=z_{0}$ because $q^{\prime} \circ \phi\left(\psi^{-1}\left(t_{0}\right)\right)$ is in the fiber of $g$ over $u_{0}$.

By item (ii), $t_{0}^{\prime} \in \psi^{-1}\left(t_{0}\right)$. Then $q^{\prime} \circ \phi\left(t_{0}^{\prime}\right)=z_{0}$, and by item (iii) we have $y_{0}=\phi\left(t_{0}^{\prime}\right)$, then $q^{\prime}\left(y_{0}\right)=q^{\prime}\left(\phi\left(t_{0}^{\prime}\right)\right)=z_{0}$ and the claim is proved. 
In Section 2 we will construct this family under some assumptions on $A$ (Proposition 2.5), and in Section 3 we will show how to use that to construct a family for any $A$. Note that because of Remark 1 we can assume $h^{1}(A)>0$.

\section{A particular case.}

Given a rank one torsion-free sheaf $A$ on an integral curve lying on a surface $S$, we define another sheaf $A^{*}$ that is going to be some sort of dual. Let $j$ be the inclusion of the curve $C$ in the surface $S$. We define $A^{*}$ as follows:

$$
A^{*}=\operatorname{Ext}^{1}\left(j_{*} A, \omega_{S}\right) .
$$

The operation $A \rightarrow A^{*}$ is a contravariant functor. Note that the support of $A^{*}$ is $C$. It will be clear from the context when we are referring to $A^{*}$ as a torsion sheaf on $S$ or as a sheaf on $C$. In the case in which $A$ is a line bundle, then $A^{*}=A^{\vee} \otimes \omega_{C}$. Now we prove some properties of this "dual".

Lemma 2.1. Let $A$ be a rank one torsion-free sheaf on an integral curve lying on a surface. Then $A^{* *}=A$.

Proof. First observe that if $L$ is a line bundle on $C$, then $(A \otimes L)^{*} \cong A^{*} \otimes L^{\vee}$. To see this, take an injective resolution of $\omega_{S}$

$$
0 \rightarrow \omega_{S} \rightarrow \mathcal{I}_{0} \rightarrow \mathcal{I}_{1} \rightarrow \cdots
$$

Now we use this resolution to calculate the Ext sheaf.

$$
\begin{gathered}
\operatorname{Ext}^{1}\left(A \otimes L, \omega_{S}\right)=h^{1}\left(\operatorname{Hom}\left(A \otimes L, \mathcal{I}_{\bullet}\right)\right)=h^{1}\left(L^{\vee} \otimes \operatorname{Hom}\left(A, \mathcal{I}_{\bullet}\right)\right)= \\
=L^{\vee} \otimes h^{1}\left(\operatorname{Hom}\left(A, \mathcal{I}_{\bullet}\right)\right)=L^{\vee} \otimes \operatorname{Ext}^{1}\left(A, \omega_{S}\right) .
\end{gathered}
$$

The third equality follows from the fact that $\operatorname{Hom}\left(A, \mathcal{I}_{\bullet}\right)$ is supported on the curve and $L^{\vee}$ is locally free

It follows that $(L \otimes A)^{* *} \cong L \otimes A^{* *}$, and then proving the lemma for $A$ is equivalent to proving it for $L \otimes A$. Multiplying with an appropriate very ample line bundle, we can assume that $A$ is generated by global sections. Then we have an exact sequence

$$
0 \rightarrow E \rightarrow V \otimes \mathcal{O}_{S} \rightarrow A \rightarrow 0,
$$

where $V=H^{0}(A)$. The following lemma proves that $E$ is locally free. 
Lemma 2.2. Let $M$ be a torsion-free sheaf on an integral curve $C$ that lies on a smooth surface $S$. Let $j: C \rightarrow S$ be the inclusion. Let $F$ be a locally free sheaf on the surface. Let $f: F \rightarrow j_{*} M$ be a surjection. Then the elementary transformation $F^{\prime}$ of $F$, defined as the kernel of $f$

$$
0 \rightarrow F^{\prime} \rightarrow F \stackrel{f}{\rightarrow} j_{*} M \rightarrow 0
$$

is a locally free sheaf.

Proof. $M$ is torsion-free sheaf on $C$, and then $j_{*} M$ has depth at least one, and because $S$ is smooth of dimension 2, this implies that the projective dimension of $j_{*} M$ is at most one $\left(E x t^{i}\left(j_{*} M, \mathcal{O}_{S}\right)=0\right.$ for $\left.i \geq 2\right)$. Now $\operatorname{Ext}^{i}\left(F, \mathcal{O}_{S}\right)=0$ for $i \geq 1$ because $F$ is locally free, and then from the exact sequence $(2.2)$, we get

$$
0 \rightarrow \operatorname{Ext}^{i}\left(F^{\prime}, \mathcal{O}_{S}\right) \rightarrow \operatorname{Ext}^{i+1}\left(j_{*} M, \mathcal{O}_{S}\right) \rightarrow 0, \quad i \geq 1,
$$

and then $\operatorname{Ext} t^{i}\left(F^{\prime}, \mathcal{O}_{S}\right)=0$ for $i \geq 1$, and this implies that $F^{\prime}$ is locally free.

In particular, $E^{\vee \vee}=E$. Applying the functor $\operatorname{Hom}\left(\cdot, \omega_{S}\right)$ twice to the sequence (2.1), we get

$$
0 \rightarrow E \rightarrow V \otimes \mathcal{O}_{S} \rightarrow A^{* *} \rightarrow 0 .
$$

Comparing with (2.1) we get the result (because the map on the left is the same for both sequences).

Lemma 2.3. $\operatorname{Ext}^{1}\left(A, \omega_{S}\right) \cong H^{0}\left(A^{*}\right)$, and this is dual to $H^{1}(A)$.

Proof. The local to global spectral sequence for Ext gives the following exact sequence

$$
0 \rightarrow H^{1}\left(H o m\left(A, \omega_{S}\right)\right) \rightarrow \operatorname{Ext}^{1}\left(A, \omega_{S}\right) \rightarrow H^{0}\left(A^{*}\right) \rightarrow H^{2}\left(H o m\left(A, \omega_{S}\right)\right)
$$

But $\operatorname{Hom}\left(A, \omega_{S}\right)=0$ because $A$ is supported in $C$ and then the first and last terms in the sequence are zero and we have the desired isomorphism.

Now we will prove a lemma that we will need. The proof can also be found in $[\mathrm{O}]$, but for convenience we reproduce it here. 
Lemma 2.4. Let $E$ and $F$ be two vector bundles of rank $e$ and $f$ over a smooth variety $X$. Assume that $E^{\vee} \otimes F$ is generated by global sections. If $\phi: E \rightarrow F$ is a sheaf morphism, we define $D_{k}(\phi)$ to be the subset of $X$ where $\operatorname{rk}\left(\phi_{x}\right) \leq k$ (there is an obvious determinantal description of $D_{k}(\phi)$ that gives a scheme structure). Let $d_{k}$ be the expected dimension of $D_{k}(\phi)$

$$
d_{k}=\operatorname{dim}(X)-(e-k)(f-k) .
$$

Then there is a Zariski dense set $U$ of $\operatorname{Hom}(E, F)$ such that if $\phi \in U$, then we have that $D_{k}(\phi) \backslash D_{k-1}(\phi)$ is smooth of the expected dimension (if $d_{k}<0$ then it will be empty).

Proof. Let $M_{k}$ be the set of matrices of dimension $e \times f$ and of rank at most $k$ (there is an obvious determinantal description that gives a scheme structure to this subvariety). It is well known that the codimension of $M_{k}$ in the space of all matrices is $(e-k)(f-k)$, and that the singular locus of $M_{k}$ is $M_{k-1}$.

Now, because $E^{\vee} \otimes F$ is generated by global sections, we have a surjective morphism

$$
H^{0}\left(E^{\vee} \otimes F\right) \otimes \mathcal{O}_{X} \rightarrow E^{\vee} \otimes F
$$

that gives a morphism of maximal rank between the varieties defined as the total space of the previous vector bundles

$$
p: X \times H^{0}\left(E^{\vee} \otimes F\right) \rightarrow \mathbb{V}\left(E^{\vee} \otimes F\right) .
$$

Define $\Sigma_{k} \subset \mathrm{V}\left(E^{\vee} \otimes F\right)$ as the set such that $\operatorname{rk}\left(\phi_{x}\right) \leq k$. The fiber of $\Sigma_{k}$ over any point in $X$ is obviously $M_{k}$. Define $Z_{k}$ to be $p^{-1}\left(\Sigma_{k}\right)$. The fact that $p$ has maximal rank implies that $Z_{k}$ has codimension $(e-k)(f-k)$ in $X \times H^{0}\left(E^{\vee} \otimes F\right)$ and that the singular locus of $Z_{k}$ is $Z_{k-1}$.

Now observe that the restriction of the projection

$$
\left.q\right|_{Z_{k} \backslash Z_{k-1}}: Z_{k} \backslash Z_{k-1} \rightarrow H^{0}\left(E^{\vee} \otimes F\right)
$$

has fiber $\left.q\right|_{Z_{k} \backslash Z_{k-1}}{ }^{-1}(\phi) \cong D_{k}(\phi) \backslash D_{k-1}(\phi)$. Finally, by generic smoothness, for a general $\phi \in H^{0}\left(E^{\vee} \otimes F\right)$ this is smooth of the expected dimension (or empty).

Now we will construct the deformation of $A$ that we described in the section 1 in the particular case in which both $A$ and $A^{*}$ are generated by global sections. 
Proposition 2.5. Let $A$ be a rank one torsion-free sheaf on an integral curve $C$ lying on a surface $S$ with $h^{1}\left(\mathcal{O}_{S}\right)=0$ and $-K_{S}$ generated by global sections. Denote $j: C \hookrightarrow S$. If $A$ and $A^{*}$ are both generated by global sections, then there exists a (not necessarily complete) smooth irreducible curve $T$ and a sheaf $\mathcal{A}$ on $S \times T$ flat over $T$, such that

(a) the sheaf induced on the fiber of $S \times T \rightarrow T$ over some $t_{0} \in T$ is $j_{*} A$

(b) the sheaf $A_{t}$ induced on the fiber over any $t \in T$ with $t \neq t_{0}$ is supported on a smooth curve $C_{t}$ and it is a rank one torsion-free sheaf when considered as a sheaf on $C_{t}$

(c) $h^{0}\left(A_{t}\right) \geq h^{0}(A)$ for every $t \in T$.

Note that these are the hypothesis of Lemma 1.1 for the particular case in which both $A$ and $A^{*}$ are generated by global sections. We will lift this condition in the next section.

Proof. The fact that $A$ is generated by global sections implies that there is an exact sequence

$$
0 \rightarrow E \stackrel{f_{0}}{\rightarrow} V \otimes \mathcal{O}_{S} \rightarrow A \rightarrow 0 \quad V=H^{0}(A),
$$

with $E$ locally free (by Proposition 2.2). Taking global sections in this sequence we see that $H^{0}(E)=0$, because

$$
0 \rightarrow H^{0}(E) \rightarrow V \stackrel{\cong}{\rightarrow} H^{0}(A) \text {. }
$$

Consider a curve $T$ mapping to $\operatorname{Hom}\left(E, V \otimes \mathcal{O}_{S}\right)$ with $t_{0} \in T$ mapping to $f_{0}$ (so that item (a) is satisfied). Denote by $f_{t}$ the morphism given for $t \in T$ by this map. After shrinking $T$ we can assume that $f_{t}$ is still injective. Let $\pi_{1}$ be the projection of $S \times T$ onto the first factor and let $\mathcal{E}=\pi_{1}^{*} E$. Using the universal sheaf and morphism on $\operatorname{Hom}\left(E, V \otimes \mathcal{O}_{S}\right)$ we can construct (by pulling back to $S \times T)$ an exact sequence on $S \times T$

$$
0 \rightarrow \mathcal{E} \stackrel{f}{\rightarrow} V \otimes \mathcal{O}_{S \times T} \rightarrow \mathcal{A} \rightarrow 0
$$

that restricts for each $t$ to an exact sequence

$$
0 \rightarrow E \stackrel{f_{t}}{\rightarrow} V \otimes \mathcal{O}_{S} \rightarrow A_{t} \rightarrow 0,
$$

where $A_{t}$ is a sheaf supported in the degeneracy locus of $f_{t}$. It is clear that $\operatorname{deg}(A)=\operatorname{deg}\left(A_{t}\right)$. 
Now we are going to prove that if the curve $T$ and the mapping to $\operatorname{Hom}\left(E, V \otimes \mathcal{O}_{S}\right)$ are chosen generically, the quotient of the map gives the desired deformation.

The flatness of $\mathcal{A}$ over $T$ follows from the fact that it has a short resolution and from the local criterion of flatness (we can apply [H, III Lemma 10.3.A]).

The condition on $h^{0}\left(A_{t}\right)$ follows because $H^{0}(E)=0$ and we have a sequence

$$
0 \rightarrow H^{0}(E)=0 \rightarrow V \rightarrow H^{0}\left(A_{t}\right),
$$

and then $h^{0}(A) \leq h^{0}\left(A_{t}\right)$. This proves item (c).

Using the long exact sequence obtained by applying $\operatorname{Hom}\left(\cdot, \mathcal{O}_{S}\right)$ to $(2.3)$, and the fact that $E$ is locally free, we obtain that $\operatorname{Ext}^{i}\left(A_{t}, \mathcal{O}_{S}\right)$ vanishes for $i \geq 2$, and so the projective dimension of $A_{t}$ is 1 , and this implies that $A_{t}$, when considered as a sheaf on its support $C_{t}$, is torsion-free.

We have to prove that we can choose the curve $T$ and the map to $\operatorname{Hom}\left(E, V \otimes \mathcal{O}_{S}\right)$ such that $C_{t}$ is smooth for $t \neq t_{0}$ (here we will use that $A^{*}$ is generated by global sections).

First note that $\operatorname{Ext}^{1}\left(A, \mathcal{O}_{S}\right)$ is generated by global sections, because $\operatorname{Ext} t^{1}\left(A, \mathcal{O}_{S}\right)=A^{*} \otimes \omega_{S}^{-1}$, and both $A^{*}$ and $\omega_{S}^{-1}$ are generated by global sections. Now we see that $E^{\vee}$ is generated by global sections, because we have

$$
0 \rightarrow V^{\vee} \otimes \mathcal{O}_{S} \rightarrow E^{\vee} \rightarrow \operatorname{Ext}^{1}\left(A, \mathcal{O}_{S}\right) \rightarrow 0,
$$

$\operatorname{Ext}^{1}\left(A, \mathcal{O}_{S}\right)$ is generated by global sections and $H^{1}\left(V^{\vee} \otimes \mathcal{O}_{S}\right)=0$. Then $E^{\vee} \otimes\left(V \otimes \mathcal{O}_{S}\right)$ is generated by global sections.

Now apply Lemma 2.4 with $F=V \otimes \mathcal{O}_{S}$. Then $n=m=r+1, k=r$ and the expected dimension is 1 . And the lemma gives that for $\phi$ in a Zariski open subset of $\operatorname{Hom}\left(E, V \otimes \mathcal{O}_{S}\right)$, the degeneracy locus $D_{r}(\phi)$ of $\phi$ is smooth away from the locus $D_{r-1}(\phi)$ where $\phi$ has rank $r-1$, but again by Lemma 2.4 the locus $D_{r-1}(\phi)$ is empty. This proves item (b).

\section{General case.}

Now we don't assume that $A$ satisfies the properties of the particular case (i.e., $A$ and $A^{*}$ now might not be generated by global sections). We will find a new sheaf that satisfies those conditions. We know how to deform this new sheaf, and we will show how we can use this deformation to construct a deformation of the original $A$.

We start with a rank one torsion-free sheaf $A$ with $h^{0}(A), h^{1}(A)>0$ on an integral curve $C$ lying on a surface. First we define $A^{\prime}$ as the base point 
free part of $A$, i.e., $A^{\prime}$ is the image of the evaluation map

$$
H^{0}(A) \otimes \mathcal{O}_{C} \rightarrow A
$$

We have assumed that $h^{0}(A)>0$, and then $A^{\prime}$ is a (nonzero) rank one torsion-free sheaf. Obviously, $H^{0}(A)=H^{0}\left(A^{\prime}\right)$. We have a short exact sequence

$$
0 \rightarrow A^{\prime} \rightarrow A \rightarrow Q \rightarrow 0
$$

where $Q$ has support of dimension 0 . Now consider $A^{\prime *}$, and define $B$ to be its base point free part. We have $h^{0}\left(A^{\prime *}\right)=h^{1}\left(A^{\prime}\right)=h^{1}(A)+h^{0}(Q) \geq$ $h^{1}(A)>0$. The first equality by lemma 2.3 , and the last inequality by assumption. Then $B$ is a (nonzero) rank one torsion-free sheaf. Finally define $A^{\prime \prime}$ to be equal to $B^{*}$.

Lemma 3.1. Both $A^{\prime \prime}$ and $A^{\prime \prime *}$ are generated by global sections.

Proof. Since $B$ is the base point free part of $A^{\prime *}$, we have a sequence

$$
0 \rightarrow B \rightarrow A^{\prime *} \rightarrow R \rightarrow 0
$$

where $R$ has support of dimension zero. Applying $\operatorname{Hom}\left(\cdot, \omega_{S}\right)$ we get

$$
0 \rightarrow A^{\prime} \rightarrow B^{*}=A^{\prime \prime} \rightarrow \widetilde{R} \rightarrow 0 \quad \widetilde{R}=\operatorname{Ext}^{2}\left(R, \omega_{S}\right),
$$

whose associated cohomology long exact sequence gives

$$
0 \rightarrow H^{0}\left(A^{\prime}\right) \rightarrow H^{0}\left(B^{*}\right) \rightarrow H^{0}(\widetilde{R}) \rightarrow H^{1}\left(A^{\prime}\right) \rightarrow H^{1}\left(B^{*}\right) \rightarrow 0 .
$$

To see that $A^{\prime \prime}$ is generated by global sections, it is enough to prove that the last map is an isomorphism, because then the first three terms make a short exact sequence, and the fact that $A^{\prime}$ and $\widetilde{R}$ are generated by global sections (the first by definition, the second because its support has dimension zero) will imply that $B^{*}$ (that is equal to $A^{\prime \prime}$ by definition) is generated by global sections.

To prove that the last map is an isomorphism, we only need to show that $h^{1}\left(A^{\prime}\right)=h^{1}\left(B^{*}\right)$, and this is true because

$$
h^{1}\left(A^{\prime}\right)=h^{0}\left(A^{\prime *}\right)=h^{0}(B)=h^{0}\left(B^{* *}\right)=h^{1}\left(B^{*}\right) .
$$

The first equality is by Lemma 2.3 , the second because $B$ is the base point free part of $A^{\prime *}$, the third by Lemma 2.1 , and the last again by Lemma 2.3 . 
To see that $A^{\prime \prime *}$ is generated by global sections, note that by definition $A^{\prime \prime *}=B^{* *}=B$, and this is generated by global sections.

We started with a rank one torsion-free sheaf $A$ with $h^{0}(A)$ and $h^{1}(A)>$ 0 , and we have constructed new sheaves $A^{\prime}$ and $A^{\prime \prime}$ with (nontrivial) maps $A^{\prime} \rightarrow A$ and $A^{\prime} \rightarrow A^{\prime \prime}$. They give rise to exact sequences

$$
\begin{gathered}
0 \rightarrow A^{\prime} \rightarrow A \rightarrow Q \rightarrow 0 \\
0 \rightarrow A^{\prime} \rightarrow A^{\prime \prime} \rightarrow \widetilde{Q} \rightarrow 0 .
\end{gathered}
$$

Lemma 3.2. With the previous definitions we have $h^{0}\left(A^{\prime}\right)=h^{0}(A)$ and $h^{1}\left(A^{\prime \prime}\right)=h^{1}\left(A^{\prime}\right)$.

Proof. By construction $h^{0}\left(A^{\prime}\right)=h^{0}(A)$ and $h^{0}\left(A^{\prime \prime *}\right)=h^{0}\left(A^{\prime *}\right)$. By lemma 2.3 this last equality is equivalent to $h^{1}\left(A^{\prime \prime}\right)=h^{1}\left(A^{\prime}\right)$.

As $A^{\prime \prime}$ and $A^{\prime \prime *}$ are generated by global sections, then by Proposition 2.5 the sheaf $A^{\prime \prime}$ can be deformed in a family $A_{t}^{\prime \prime}$ in such a way that the support of a general member of the deformation is smooth. The idea now is to find (flat) deformations of $A^{\prime}$ and $A$, so that for every $t$ we still have maps like (3.1a) and (3.1b). From the existence of these maps we will be able to obtain the condition that $h^{0}\left(A_{t}\right) \geq h^{0}(A)$, then we will be able to apply Lemma 1.1 and then Theorem I will be proved. The details are in Section 4. We will start by showing how the condition on $h^{0}\left(A_{t}\right)$ is obtained, and then how we can find the deformations of $A^{\prime}$ and $A$.

Proposition 3.3. Let $A, A^{\prime}, A^{\prime \prime}$ be rank one torsion-free sheaves on an integral curve $C$. Assume that they fit into exact sequences like (3.1) and that $h^{0}\left(A^{\prime}\right)=h^{0}(A)$ and $h^{1}\left(A^{\prime}\right)=h^{1}\left(A^{\prime \prime}\right)$. Let $P$ be a curve (not necessarily complete), and let $\mathcal{A}, \mathcal{A}^{\prime}$, and $\mathcal{A}^{\prime \prime}$ be sheaves on $S \times P$, flat over $P$, inducing for each $p \in P$ rank one torsion-free sheaves $A_{p}, A_{p}^{\prime}, A_{p}^{\prime \prime}$, supported on a curve $C_{p}$ of $S$, where $A_{p_{0}}=A, A_{p_{0}}^{\prime}=A^{\prime}$, and $A_{p_{0}}^{\prime \prime}=A^{\prime \prime}$ for some $p_{0} \in P$. Assume that $h^{0}\left(A_{p}^{\prime \prime}\right) \geq h^{0}\left(A_{p_{0}}^{\prime \prime}\right)$ for all $p \in P$ and that we have short exact sequences

$$
\begin{gathered}
0 \rightarrow \mathcal{A}^{\prime} \rightarrow \mathcal{A} \rightarrow \mathcal{Q} \rightarrow 0 \\
0 \rightarrow \mathcal{A}^{\prime} \rightarrow \mathcal{A}^{\prime \prime} \rightarrow \widetilde{\mathcal{Q}} \rightarrow 0
\end{gathered}
$$


with $\mathcal{Q}$ and $\widetilde{\mathcal{Q}}$ flat over $P$ (i.e., the induced sheaves $Q_{p}, \widetilde{Q}_{p}$ have constant length, equal to $l(Q)$ and $l(\widetilde{Q})$ respectively).

Then we have $h^{0}\left(A_{p}\right) \geq h^{0}\left(A_{p_{0}}\right)$ for all $p \in P$.

Proof. For each $p \in P$ we have sequences

$$
\begin{aligned}
& 0 \rightarrow A_{p}^{\prime} \rightarrow A_{p} \rightarrow Q_{p} \rightarrow 0 \\
& 0 \rightarrow A_{p}^{\prime} \rightarrow A_{p}^{\prime \prime} \rightarrow \widetilde{Q}_{p} \rightarrow 0 .
\end{aligned}
$$

The maps on the left are injective because they are nonzero and the sheaves have rank one and are torsion-free. Using the associated long exact sequences and the hypothesis we have

$$
h^{0}\left(A_{p}\right) \geq h^{0}\left(A_{p}^{\prime}\right) \geq h^{0}\left(A_{p}^{\prime \prime}\right)-l\left(\widetilde{Q}_{p}\right) \geq h^{0}\left(A^{\prime \prime}\right)-l(\widetilde{Q})=h^{0}\left(A^{\prime}\right)=h^{0}(A) .
$$

It only remains to prove that those sheaves can be "deformed along", and that those deformations are flat, i.e. that given $A, A^{\prime}$ and $A^{\prime \prime}$ we can construct $\mathcal{A}^{\prime}$ and $\mathcal{A}^{\prime \prime}$. This is proved in the following propositions.

Proposition 3.4. Let $L$ and $M$ be rank one torsion-free sheaves on an integral curve $C$ that lies on a surface $S$. Assume we have a short exact sequence

$$
0 \rightarrow L \rightarrow M \rightarrow Q \rightarrow 0
$$

Assume furthermore that we are given a sheaf $\mathcal{M}$ on $S \times P$ (where $P$ is a connected but not necessarily irreducible curve) that is a deformation of $M$, flat over $P$. I.e., $\left.\mathcal{M}\right|_{p_{0}} \cong M$ for some $p_{0} \in P$, and for all $p \in P$ we have that $M_{p}=\left.\mathcal{M}\right|_{p}$ are torsion-free sheaves on $C_{p}$, where $C_{p}$ is a curve on $S$.

Then, there is a connected curve $P^{\prime}$ with a map $f: P^{\prime} \rightarrow P$ and a sheaf $\mathcal{L}^{\prime}$ over $S \times P^{\prime}$ with the following properties:

One irreducible component of $P^{\prime}$ is a finite cover of $P$ and the rest of the components map to $p_{0} \in P$. The sheaf $\mathcal{L}^{\prime}$ is a deformation of $L$, in the sense that $\left.\mathcal{L}^{\prime}\right|_{p_{0}^{\prime}} \cong L$ for some $p_{0}^{\prime} \in P^{\prime}$ mapping to $p_{0} \in P$, the sheaf $\mathcal{L}^{\prime}$ is flat over $P^{\prime}$ and induces rank one torsion-free sheaves on the fibers over $P^{\prime}$. And if we define $\mathcal{M}^{\prime}$ to be the pullback of $\mathcal{M}$ to $S \times P^{\prime}$, there exists an exact sequence

$$
0 \rightarrow \mathcal{L}^{\prime} \rightarrow \mathcal{M}^{\prime} \rightarrow \mathcal{Q}^{\prime} \rightarrow 0
$$


inducing short exact sequences

$$
0 \rightarrow L_{p^{\prime}}^{\prime} \rightarrow M_{p^{\prime}}^{\prime} \rightarrow Q_{p^{\prime}}^{\prime} \rightarrow 0
$$

for every $p^{\prime} \in P^{\prime}$.

Proof. If the support of $Q$ were in the smooth part of the curve, we would have $M \cong L \otimes \mathcal{O}_{C}(D)$, with $D$ an effective divisor of degree $l(Q)$. Then, if we are given a deformation $M_{p}$ of $M$, we only need to find a deformation $D_{p}$ of the effective divisor $D$, with the only condition that $D_{p}$ is an effective divisor on $C_{p}$, with degree $l(Q)$. This can easily be done if we are in the analytic category. In general we might need to do a base change of the parametrizing curve $P$ and we will obtain a finite cover $P^{\prime}$ of $P$ (What we are doing is moving a dimension zero and length $l(Q)$ subscheme of $S$, with the only restriction that for each $p$ the corresponding scheme is in $C_{p}$ ). Then we only need to define $L_{p^{\prime}}=M_{p^{\prime}} \otimes \mathcal{O}_{C_{p^{\prime}}}\left(-D_{p^{\prime}}\right)$ and the proposition would be proved (with $P^{\prime}$ a finite cover of $P$ ).

To be able to apply this, we will have to make first a deformation of $L$, keeping $M$ fixed, until we get $Q$ to be supported in the smooth part of $C$ (the curve $C$ also remains fixed in this deformation). This is the reason for the need of the curve $P^{\prime}$ with some irreducible components mapping to $p_{0}$.

We will prove this by induction on the length of the intersection of the support of $Q$ and the singular part of $C$.

Lemma 3.5. Let $L$ and $M$ be rank one torsion-free sheaves on an integral curve $C$ that lies on a surface $S$. Assume we have a short exact sequence

$$
0 \rightarrow L \rightarrow M \rightarrow Q \rightarrow 0 .
$$

Assume that $Q=R \oplus Q^{\prime}$ where $Q^{\prime}$ has length $l(Q)-1$ and it is supported in the smooth part of $C$, and $R$ has length one as it is supported in a singular point of $C$ ("the length of the intersection of the support of $Q$ and the singular part of $C$ is one").

Then there is a flat deformation $L_{y}$ of $L$ parametrized by a connected curve $Y$ (it might not be irreducible) such that $L_{y_{0}}=L$ for some $y_{0} \in Y$ and for every $y \in Y$ there is an exact sequence

$$
0 \rightarrow L_{y} \rightarrow M \rightarrow Q_{y} \rightarrow 0
$$

and there is some $y_{1} \in Y$ such that the support of $Q_{y_{1}}$ is in the smooth part of $C$. 
Proof. In this situation, the exact sequence (3.2) gives rise to another exact sequence

$$
0 \rightarrow L \otimes I_{Z}^{\vee} \rightarrow M \rightarrow R \rightarrow 0
$$

where the map on the right is the composition of $M \rightarrow Q$ and the projection $Q \rightarrow R$, and we denote by $I_{Z}$ the ideal sheaf of the support $Z$ of $Q^{\prime}$. Because $Z$ is in the smooth part of $C, I_{Z}$ is an invertible sheaf. Note that $Q^{\prime}$ is the quotient of $\mathcal{O}_{C}$ by this ideal sheaf. Define $\widehat{L}$ to be $L \otimes I_{Z}^{\vee}$. If we know how to make a flat deformation $\widehat{L}_{y}$ of $\widehat{L}$ so that the quotient $R_{y}$ is supported in the smooth part of $C$ for some $y_{1} \in Y$, then we can construct a deformation $L_{y}$ of $L$ defined as

$$
L_{y}=\widehat{L}_{y} \otimes I_{Z}
$$

Note that this deformation is also flat. The cokernel $Q_{y}$ of $L_{y} \rightarrow M$ is supported in the smooth part of $C$ for the points $y \in Y$ for which $R_{y}$ is supported in the smooth part of $C$.

This shows that to prove the lemma we can assume that $Q$ has length one and its support is a singular point of $C$, i.e. $Q=\mathcal{O}_{x}$, where $x$ is a singular point of $C$.

Consider the scheme $\operatorname{Quot}^{1}(M)$ representing the functor of quotients of $M$ of length 1. If the support $x$ of the quotient $Q$ is in the smooth part of $C$, then there is only one surjective map (up to scalar) because $\operatorname{dim} \operatorname{Hom}(M, Q)=1$, whose kernel is $M \otimes \mathcal{O}_{C}(-x)$.

If $x$ is in the singular part, then in general $\operatorname{dim} \operatorname{Hom}(M, Q)>1$, and the quotients are parametrized by $\mathbb{P} \operatorname{Hom}(M, Q)$ (the universal bundle is flat over $\mathbb{P} \operatorname{Hom}(M, Q))$. We want to show that $\operatorname{Quot}^{1}(M)$ is connected by constructing a flat family of quotients $M \rightarrow \widetilde{Q}_{\tilde{c}}$ (the family $\widetilde{Q}_{\tilde{c}}$ will be parametrized by an open set of the normalization $\widetilde{C}$ of $C$ ) such that for a general $\tilde{c}$ the support of $\widetilde{Q}_{\tilde{c}}$ is in the smooth part of $C$, and for some point $\tilde{c}_{0}$ the support of $\widetilde{Q}_{\tilde{c}_{0}}$ is a singular point of $C$.

Consider the normalization $\widetilde{C}$ of $C$, and let $F$ be an open set of $\widetilde{C}$

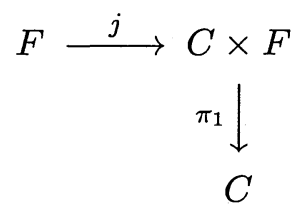

Where $\pi_{1}$ is the projection to the first factor and $j=(\nu, i)$, the morphism $\nu: F \hookrightarrow \widetilde{C} \rightarrow C$ being the restriction to $F$ of the normalization map and $i$ the identity map. Note that $j$ is a closed immersion, and its image is just $C \times{ }_{C} F \cong F$. 
Let $\tilde{c}_{0}$ be a point of $\widetilde{C}$ in $\nu^{-1}(x)$ (the family is going to be parametrized by an open neighborhood $F$ of $\tilde{c}_{0}$ ). We have to construct a surjection of $\widetilde{\mathcal{M}}=\pi_{1}^{*} M$ onto $\widetilde{\mathcal{Q}}=j_{*} \mathcal{O}_{F}$. Note that $\left.\widetilde{\mathcal{Q}}\right|_{C \times \bar{c}}=\widetilde{Q}_{\bar{c}} \cong \mathcal{O}_{\nu(\tilde{c})}$ and that $\widetilde{\mathcal{Q}}$ is flat over $F$.

Now, to define that quotient, it is enough to define it in the restriction to the image of $j$ (because this is exactly the support of $\widetilde{\mathcal{Q}}$ ). So the map we have to define is

$$
j^{*} \widetilde{\mathcal{M}} \rightarrow \mathcal{O}_{F} .
$$

But $j^{*} \widetilde{\mathcal{M}}=\nu^{*} M$ is a rank one sheaf on the smooth curve $F$, so it is the direct sum of a line bundle and a torsion part $T$. Shrinking $F$ if necessary, the line bundle part is isomorphic to $\mathcal{O}_{F}$, and we have

$$
j^{*} \widetilde{\mathcal{M}} \cong T \oplus \mathcal{O}_{F}
$$

and then to define the quotient we just take an isomorphism in the torsionfree part. This finishes the proof of the lemma.

Now we go to the general case: the intersection of the support of $Q$ with the singular part of $C$ has length $n$. We are going to see how this can be reduced to the case $n=1$.

Take a surjection from $Q$ to a sheaf $Q^{\prime}$ of length $n-1$, such that $Q$ is isomorphic to $Q^{\prime}$ at the smooth points. The kernel $R$ of this surjection will have length 1 , and will be supported in a singular point of $C$. It is isomorphic to $\mathcal{O}_{x}$, for some singular point $x$. We have a diagram

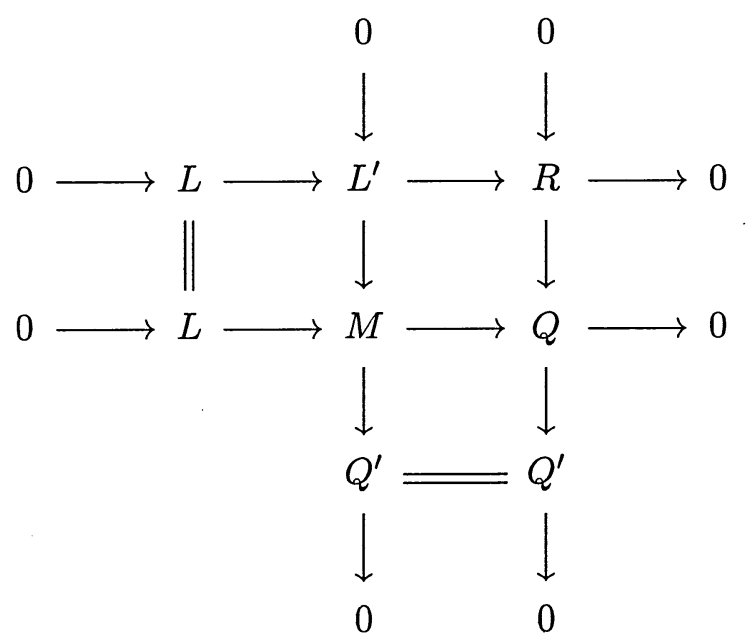


Observe that $L, L^{\prime}$ and $R$ satisfy the hypothesis of Lemma 3.5, so we can find deformations $L_{y}, R_{y}$ (parametrized by some curve $Y$ and with $L_{y_{0}}=L$ and $R_{y_{0}}=R$ for some $y_{0} \in Y$ ) such that for some $y_{1} \in Y$ we have that the support of the corresponding sheaf $R_{y_{1}}$ is a smooth point of $C$. All the maps of the previous diagram can be deformed along. To do this, we change $L$ by $L_{y}, R$ will be deformed to $R_{y}$ and $L^{\prime}$ is kept constant. Then $Q$ is deformed to a family $Q_{y}$ defined as $M / L_{y}$. The cokernel of $R_{y} \rightarrow Q_{y}$ will be $Q_{y} / R_{y}=M / L^{\prime}=Q^{\prime}$, and hence we keep it constant. Then for each $y$ we still have a commutative diagram, and furthermore it is easy to see that all deformations are flat (note that $R_{y}$ is a flat deformation and $Q^{\prime}$ is kept constant, and then $Q_{y}$ is a flat deformation). An important point is that $M$ remains fixed, and the injection $L \rightarrow M$ is deformed to $L_{y} \rightarrow M$.

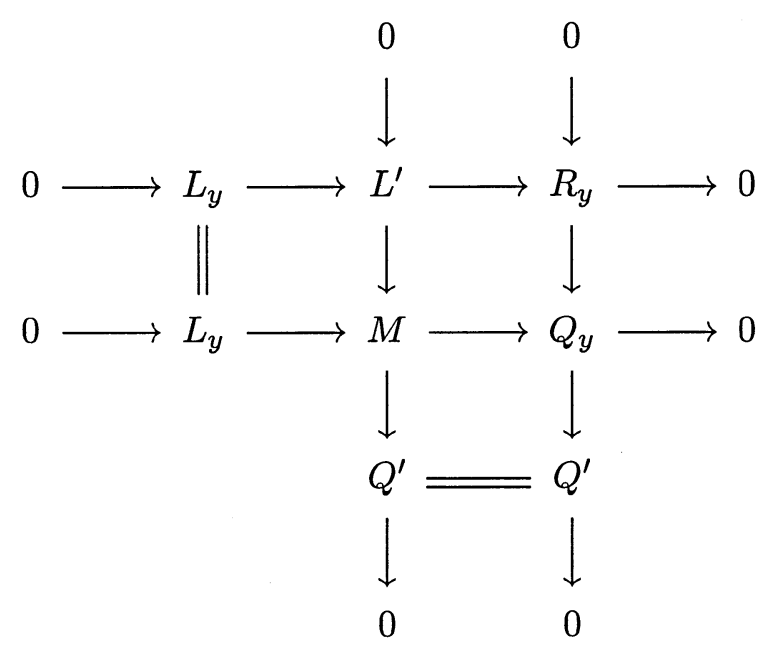

For $y_{1}$ we have that the length of the intersection of the support of $Q_{y_{1}}$ with the singular part of $C$ is $n-1$. We repeat the process (starting now with $L_{y_{1}}, M$ and $Q_{y_{1}}$ ), until all the points of the support of $Q$ are moved to the smooth part of $C$. This finishes the proof of the proposition.

The following proposition is similar to Proposition 3.4, but now the roles of $L$ and $M$ are changed: we are given a deformation of $L$ and we have to deform $M$ along.

Proposition 3.6. Let $L$ and $M$ be rank one torsion-free sheaves on an integral curve $C$ that lies on a surface $S$. Assume we have a short exact sequence

$$
0 \rightarrow L \rightarrow M \rightarrow Q \rightarrow 0
$$


Assume furthermore that we are given a sheaf $\mathcal{L}$ on $S \times P($ where $P$ is a connected but not necessarily irreducible curve) that is a deformation of $L$, flat over $P$, i.e., $\left.\mathcal{L}\right|_{p_{0}} \cong L$ for some $p_{0} \in P$, and for all $p \in P$, we have that $L_{p}=\left.\mathcal{L}\right|_{p}$ are torsion-free sheaves on $C_{p}$, where $C_{p}$ is a curve on $S$.

Then, there is a connected curve $P^{\prime}$ with a map $f: P^{\prime} \rightarrow P$ and a sheaf $\mathcal{M}^{\prime}$ over $S \times P^{\prime}$ with the following properties:

One irreducible component of $P^{\prime}$ is a finite cover of $P$ and the rest of the components map to $p_{0} \in P$. The sheaf $\mathcal{M}^{\prime}$ is a deformation of $M$, in the sense that $\left.\mathcal{M}^{\prime}\right|_{p_{0}^{\prime}} \cong M$ for some $p_{0}^{\prime} \in P^{\prime}$ mapping to $p_{0} \in P$, the sheaf $\mathcal{M}^{\prime}$ is flat over $P^{\prime}$ and induces rank one torsion-free sheaves on the fibers over $P^{\prime}$. And if we define $\mathcal{L}^{\prime}$ to be the pullback of $\mathcal{L}$ to $S \times P^{\prime}$, there exists an exact sequence

$$
0 \rightarrow \mathcal{L}^{\prime} \rightarrow \mathcal{M}^{\prime} \rightarrow \mathcal{Q}^{\prime} \rightarrow 0
$$

inducing short exact sequences

$$
0 \rightarrow L_{p^{\prime}}^{\prime} \rightarrow M_{p^{\prime}}^{\prime} \rightarrow Q_{p^{\prime}}^{\prime} \rightarrow 0
$$

for every $p^{\prime} \in P^{\prime}$.

Proof. The proof is very similar to the proof of Proposition 3.4. Again we start by observing that if the support of $Q$ were in the smooth part of the curve, we would have $M \cong L \otimes \mathcal{O}_{C}(D)$, with $D$ an effective divisor. Then if we are given a flat deformation $L_{p}$ of $L$, we find a deformation $D_{p}$ of $D$ as in the first part, and the proposition would be proved. So again we need a lemma that deforms $Q$ so that its support is in the smooth part of $C$.

Lemma 3.7. Let $L$ and $M$ be rank one torsion-free sheaves on an integral curve $C$ that lies on a surface $S$. Assume we have a short exact sequence

$$
0 \rightarrow L \rightarrow M \rightarrow Q \rightarrow 0 .
$$

Assume that the part of $Q$ with support in the smooth part of $C$ has length $l(Q)-1$, i.e., $Q=R \oplus Q^{\prime}$, where $R$ has length one and is supported in a singular point of $C$ and $Q^{\prime}$ has length $l(Q)-1$ and is supported in the smooth part of $C$. Then there is a flat deformation $M_{y}$ of $M$ parametrized by a curve $Y$, such that for every $y \in Y$ there is an exact sequence

$$
0 \rightarrow L \rightarrow M_{y} \rightarrow Q_{y} \rightarrow 0
$$

with $M_{y}$ a torsion-free sheaf, and there is some $y_{1} \in Y$ such that the support of $Q_{y_{1}}$ is in the smooth part of $C$. 
Proof. Arguing as in the proof of Lemma 3.5, we see that it is enough to prove the case $l(Q)=1$, and $Q=\mathcal{O}_{x}$ for $x$ a singular point of $C$, then we can assume that the extension of the hypothesis of the lemma is

$$
0 \rightarrow L \rightarrow M \rightarrow \mathcal{O}_{x} \rightarrow 0 .
$$

Now we will consider all extensions of $\mathcal{O}_{x}$ (for $x$ any point in $C$ ) by $L$. If $x$ is a smooth point, then there is only one extension that is not trivial (up to equivalence)

$$
0 \rightarrow L \rightarrow M \cong L \otimes \mathcal{O}_{C}(x) \rightarrow \mathcal{O}_{x} \rightarrow 0 .
$$

All these extensions are then parametrized by the smooth part of $C$.

But if $x$ is a singular point, we could have more extensions, because in general $s=\operatorname{dim} \operatorname{Ext}^{1}\left(\mathcal{O}_{x}, L\right)>1$. They will be parametrized by a projective space $\mathbb{P}^{s-1}$. We call this space $E_{x}$. Note that there is a universal extension on $C \times E_{x}$ that is flat over $E_{x}$. We denote by $e_{1}$ the point in $E_{x}$ corresponding to the extension (3.5).

Assume that $\widetilde{Q}_{\tilde{c}}$ is a family of torsion sheaves on $C$ with length 1 , parametrized by a curve $F$ such that for a general point $\tilde{c} \in F$ of the parametrizing curve the support of $\widetilde{Q}_{\tilde{c}}$ in $C$ is a smooth point, and for a special point $\tilde{c}_{0} \in F$ the support of $\widetilde{Q}_{\tilde{c}_{0}}$ is a singular point. Now assume that we can construct a flat family (parametrized by $F$ ) of nontrivial extensions of $\widetilde{Q}_{\tilde{c}}$ by $L$. The extension corresponding to $\tilde{c}_{0}$ gives a point $e_{2}$ in $E_{x}$. The space $E_{x}$ is a projective space, thus connected, and then there is a curve containing $e_{1}$ and $e_{2}$. Using this curve (together with the universal extension for $E_{x}$ ) and the curve $F$ (together with the family of extensions that it parametrizes) we construct the curve $Y$ that proves the lemma.

Now we need to construct $F$. As in the proof of Lemma 3.5, the parametrizing curve $F$ will be an affine neighborhood of $\tilde{c}_{0}$ in the normalization $\widetilde{C}$ of $C$, where $\tilde{c}_{0}$ is a point that maps to the singular point $x$ of $C$. Consider again the diagram (3.3) of the proof of Lemma 3.5. The family will be given by an extension of $\widetilde{\mathcal{Q}}=j_{*} \mathcal{O}_{F}$ by $\widetilde{\mathcal{L}}=\pi_{1}^{*} L$ on $C \times F$. These extensions are parametrized by the group $\operatorname{Ext}^{1}(\widetilde{\mathcal{Q}}, \widetilde{\mathcal{L}})$. The following lemma gives information about this group and relates this extension with the extensions that we get after restriction for each slice $C \times \tilde{c}$. We will call $\widetilde{Q}_{\tilde{c}}$ and $\widetilde{L}_{\tilde{c}}$ the restrictions of $\widetilde{\mathcal{Q}}$ and $\widetilde{\mathcal{L}}$ to the slice $C \times \tilde{c}$. Note that the restriction $\widetilde{L}_{\tilde{c}}$ is isomorphic to $L$.

Lemma 3.8. With the previous notation, we have

1) $\operatorname{Ext}^{1}(\widetilde{\mathcal{Q}}, \widetilde{\mathcal{L}}) \cong H^{0}\left(\operatorname{Ext}^{1}(\widetilde{\mathcal{Q}}, \widetilde{\mathcal{L}})\right)$ 
2) $\operatorname{Ext}^{1}(\widetilde{\mathcal{Q}}, \widetilde{\mathcal{L}})$ has rank zero outside of the support of $\widetilde{\mathcal{Q}}$, and rank 1 on the smooth points of the support of $\widetilde{\mathcal{Q}}$

3) Let I be the ideal sheaf corresponding to a slice $C \times \tilde{c}$. Then the natural map

$$
\operatorname{Ext}_{\mathcal{O}_{C \times F}}^{1}(\widetilde{\mathcal{Q}}, \widetilde{\mathcal{L}}) \otimes \mathcal{O}_{C \times F} / I \rightarrow \operatorname{Ext}_{\mathcal{O}_{C \times \bar{c}}}^{1}\left(\widetilde{Q}_{\tilde{c}}, \widetilde{L}_{\tilde{c}}\right)
$$

is injective.

Proof. Item 1 follows from the fact that $\operatorname{Hom}(\widetilde{\mathcal{Q}}, \widetilde{\mathcal{L}})=0$ and the exact sequence

$$
0 \rightarrow H^{1}(\operatorname{Hom}(\widetilde{\mathcal{Q}}, \widetilde{\mathcal{L}})) \rightarrow \operatorname{Ext}^{1}(\widetilde{\mathcal{Q}}, \widetilde{\mathcal{L}}) \rightarrow H^{0}\left(\operatorname{Ext}^{1}(\widetilde{\mathcal{Q}}, \widetilde{\mathcal{L}})\right) \rightarrow H^{2}(\operatorname{Hom}(\widetilde{\mathcal{Q}}, \widetilde{\mathcal{L}}))
$$

To prove item 2 note that the stalk of $\operatorname{Ext}^{1}(\widetilde{\mathcal{Q}}, \widetilde{\mathcal{L}})$ at a point $p$ is isomorphic to $\operatorname{Ext}^{1}(R / I, R)$, where $R$ is the local ring at the point $p$, and $I$ is the ideal defining the support or $\widetilde{\mathcal{Q}}$. The ideal $I$ is principal if the point $p$ is smooth, then $R / I$ has a free resolution

$$
0 \rightarrow I \rightarrow R \rightarrow R / I \rightarrow 0
$$

and it follows that $\operatorname{Ext}^{1}(R / I, R) \cong R / I$.

For item 3 , consider the exact sequence

$$
0 \rightarrow \widetilde{\mathcal{Q}} \stackrel{\cdot f}{\rightarrow} \widetilde{\mathcal{Q}} \rightarrow \widetilde{Q}_{\tilde{c}} \rightarrow 0
$$

where the first map is multiplication by the local equation $f$ of the slice $C \times \tilde{c}$. Applying $\operatorname{Hom}\left(\cdot, \widetilde{L}_{\bar{c}}\right)$ we get

$$
\operatorname{Hom}\left(\widetilde{\mathcal{Q}}, \widetilde{L}_{\tilde{c}}\right)=0 \rightarrow \operatorname{Ext}^{1}\left(\widetilde{Q}_{\tilde{c}}, \widetilde{L}_{\tilde{c}}\right) \rightarrow \operatorname{Ext}^{1}\left(\widetilde{\mathcal{Q}}, \widetilde{L}_{\tilde{c}}\right) \rightarrow \operatorname{Ext}^{1}\left(\widetilde{\mathcal{Q}}, \widetilde{L}_{\tilde{c}}\right),
$$

but the last map is zero. To see this, take a locally free resolution of $\widetilde{\mathcal{Q}}$. The map induced on the resolution by the multiplication with the equation $f$ is just multiplication by the same $f$ on each term

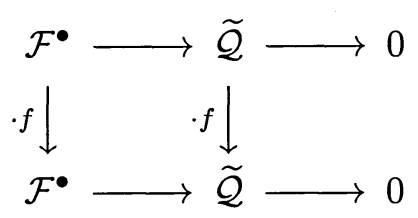

A local section of the sheaf $\operatorname{Ext}\left(\widetilde{\mathcal{Q}}^{i}, \widetilde{L}_{\tilde{c}}\right)$ is represented by some local section $\varphi(\cdot)$ of $\operatorname{Hom}\left(\mathcal{F}^{i}, \widetilde{L}_{\tilde{c}}\right)$, and the endomorphism induced by multiplication by 
$f$ on $\operatorname{Ext}^{i}\left(\widetilde{\mathcal{Q}}, \widetilde{L}_{\tilde{c}}\right)$ is given by precomposition with multiplication $\varphi(f \cdot)$, but $\varphi$ is a morphism of sheaves of modules and then this is equal to $f \varphi(\cdot)$, and this is equal to zero because $f \widetilde{L}_{\tilde{c}}=0$. Then we have that

$$
\operatorname{Ext}^{1}\left(\widetilde{Q}_{\tilde{c}}, \widetilde{L}_{\tilde{c}}\right) \cong \operatorname{Ext}^{1}\left(\widetilde{\mathcal{Q}}, \widetilde{L}_{\tilde{c}}\right) .
$$

Taking the exact sequence

$$
0 \rightarrow \widetilde{\mathcal{L}} \stackrel{\cdot f}{\rightarrow} \widetilde{\mathcal{L}} \rightarrow \widetilde{L}_{\tilde{c}} \rightarrow 0
$$

and applying $\operatorname{Hom}(\widetilde{\mathcal{Q}}, \cdot)$ we get

$$
\operatorname{Ext}^{1}(\widetilde{\mathcal{Q}}, \widetilde{\mathcal{L}}) \stackrel{\cdot f}{\rightarrow} \operatorname{Ext}^{1}(\widetilde{\mathcal{Q}}, \widetilde{\mathcal{L}}) \rightarrow \operatorname{Ext}^{1}\left(\widetilde{\mathcal{Q}}, \widetilde{L}_{\tilde{c}}\right)
$$

and using this and the isomorphism (3.6) we have an injection

$$
\operatorname{Ext}^{1}(\widetilde{\mathcal{Q}}, \widetilde{\mathcal{L}}) \otimes \mathcal{O}_{C \times F} / I \cong \operatorname{Ext}^{1}(\widetilde{\mathcal{Q}}, \widetilde{\mathcal{L}}) /\left(f \cdot \operatorname{Ext}^{1}(\widetilde{\mathcal{Q}}, \widetilde{\mathcal{L}})\right) \hookrightarrow \operatorname{Ext}^{1}\left(\widetilde{Q}_{\tilde{c}}, \widetilde{L}_{\tilde{c}}\right) .
$$

Now we are going to construct the family of extensions. By item 2 of the lemma the sheaf $\mathcal{E}=\operatorname{Ext}^{1}(\widetilde{\mathcal{Q}}, \widetilde{\mathcal{L}})$ is isomorphic to $\mathcal{O}_{X} \oplus T(\mathcal{E})$ (shrinking $F$ if necessary) where $X$ is the support of $\widetilde{\mathcal{Q}}$ and $T(\mathcal{E})$ is the torsion part. Take a nonvanishing section of the torsion-free part, and by item 1 this gives a nonzero element $\psi$ of $\operatorname{Ext}^{1}(\widetilde{\mathcal{Q}}, \widetilde{\mathcal{L}})$. This element gives a nontrivial extension

$$
0 \rightarrow \widetilde{\mathcal{L}} \rightarrow \widetilde{\mathcal{M}} \rightarrow \widetilde{\mathcal{Q}} \rightarrow 0
$$

Observe that $\widetilde{\mathcal{M}}$ is flat over the base, because both $\widetilde{\mathcal{L}}$ and $\widetilde{\mathcal{Q}}$ are flat.

By items 3 and 1 we have that the image of $\psi$ under the restriction map

$$
\operatorname{Ext}^{1}(\widetilde{\mathcal{Q}}, \widetilde{\mathcal{L}}) \rightarrow \operatorname{Ext}^{1}\left(\widetilde{Q}_{\tilde{c}}, L\right)
$$

is nonzero for any $\tilde{c}$ (recall that $\widetilde{L}_{\tilde{c}}=L$ for all $\tilde{c}$ ), and this means that the extensions that we obtain after restriction to the corresponding slices

$$
0 \rightarrow L \rightarrow \widetilde{M}_{\tilde{c}} \rightarrow \widetilde{Q}_{\tilde{c}} \rightarrow 0
$$

are non trivial. Furthermore $\widetilde{M}_{\tilde{c}}$ is torsion-free. To prove this claim, let $T\left(\widetilde{M}_{\tilde{c}}\right)$ be the torsion part of $\widetilde{M}_{\tilde{c}}$. The map $L \rightarrow T\left(\widetilde{M}_{\tilde{c}}\right)$ coming from (3.7) is zero, because $L$ is torsion-free, i.e. $T\left(\widetilde{M}_{\tilde{c}}\right)$ injects in $\widetilde{Q}_{\tilde{c}}$. Then we have

$$
\widetilde{Q}_{\tilde{c}} \cong \frac{\widetilde{M}_{\tilde{c}}}{L} \cong \frac{\widetilde{M}_{\tilde{c}} / T\left(\widetilde{M}_{\tilde{c}}\right) \oplus T\left(\widetilde{M}_{\tilde{c}}\right)}{L} \cong \frac{\widetilde{M}_{\tilde{c}} / T\left(\widetilde{M}_{\tilde{c}}\right)}{L} \oplus T\left(\widetilde{M}_{\tilde{c}}\right)
$$


$\widetilde{Q}_{\bar{c}}$ doesn't decompose as the direct sum of two sheaves, and then one of these summands must be zero. The first summand cannot be zero, because this would imply that $L \cong \widetilde{M}_{\tilde{c}} / T\left(\widetilde{M}_{\tilde{c}}\right)$ and then $\widetilde{M}_{\tilde{c}} \cong L \oplus \widetilde{Q}_{\tilde{c}}$, contradicting the hypothesis that the extension is not trivial. Then we must have $T\left(\widetilde{M}_{\tilde{c}}\right)=0$, and the claim is proved.

Now we are going to consider the general case, in which the part of $Q$ supported in singular points has length $n$. We are going to see that this can be reduced to the case $n=1$, in a similar way to proposition 3.4 .

Let $R=\mathcal{O}_{x}$, where $x$ is a singular point in the support of $Q$, and take a surjection from $Q$ to $R$. We have a diagram

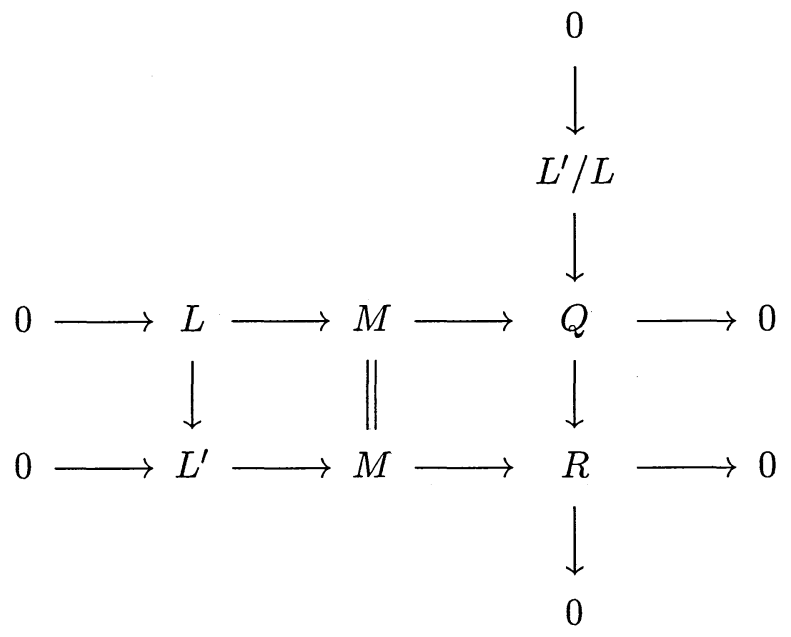

Note that $L^{\prime}, M$ and $R$ satisfy the hypothesis of Lemma 3.7, then we can find (flat) deformations $M_{y}$ and $R_{y}$ parametrized by a curve $Y$ such that for some $y_{1} \in Y$ we have that the support of the corresponding sheaf $R_{y_{1}}$ is a smooth point of $C$. All sheaves and maps can be deformed along. To do this we define $Q_{y}=M_{y} / L$ (we have $L \hookrightarrow L^{\prime} \hookrightarrow M_{y}$, thus this quotient is well defined). The kernel of $Q_{y} \rightarrow R_{y}$ is $L^{\prime} / L$. Then $Q_{y}$ is a flat deformation (being the extension of a flat deformation $R_{y}$ by a constant and hence flat 
deformation $\left.L^{\prime} / L\right)$. Then for each $y$ we have a commutative diagram

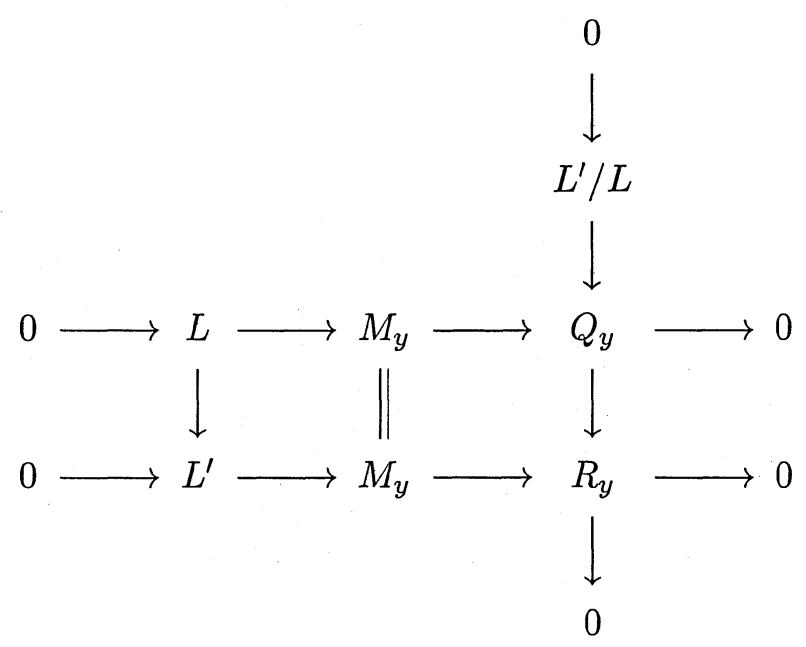

Observe that the length of the part of $Q_{y_{1}}$ supported in singular points is $n-1$, so repeating this process we can $\operatorname{deform} Q$ until its support lies in the smooth part of $C$. This finishes the proof of the proposition.

\section{Proof of Theorem I.}

In this section we will prove Theorem I:

Proof. Nonemptiness follows from the fact that the Brill-Nother loci for smooth curves is nonempty, and by upper semicontinuity of $h^{0}(\cdot)$. By Remark 1 we can assume $r>d-p_{a}$. We will prove Theorem I by applying Lemma 1.1.

We start with a rank one torsion-free sheaf $A$ corresponding to a point in $\bar{W}_{d}^{r}, d>0, r \geq 0$, with $\rho(r, d)>0$ (recall that we are assuming $r>d-p_{a}$ ). We have $h^{0}(A), h^{1}(A)>0$. As we explained at the beginning of the section 3 , we call $A^{\prime}$ its base point free part. Then we take $B$ to be the base point free part of $A^{\prime *}$, and finally define $A^{\prime \prime}$ to be $B^{*}$.

By Lemma 3.1, $A^{\prime \prime}$ and $A^{\prime \prime *}$ are rank one locally free sheaves on $C$ generated by global sections. Then by Proposition 2.5 we find a deformation $\mathcal{A}^{\prime \prime}$ of $A^{\prime \prime}$ parametrized by a some smooth irreducible curve $T$.

The support of $\mathcal{A}^{\prime \prime}$ defines a family of curves $\mathcal{C}$ parametrized by the irreducible curve $T$. Note that $\left.\mathcal{C}\right|_{t}$ is smooth for $t \neq 0$. 
By the definition of $A^{\prime}$ and $A^{\prime \prime}$ we have exact sequences

$$
\begin{aligned}
& 0 \rightarrow A^{\prime} \rightarrow A \rightarrow Q \rightarrow 0 \\
& 0 \rightarrow A^{\prime} \rightarrow A^{\prime \prime} \rightarrow \widetilde{Q} \rightarrow 0
\end{aligned}
$$

with $h^{0}\left(A^{\prime}\right)=h^{0}(A)$ and $h^{1}\left(A^{\prime \prime}\right)=h^{1}\left(A^{\prime}\right)$ (Lemma 3.2). If we look at (4.1b) we see that we are in the situation of Proposition 3.4, with $L=A^{\prime}$, $M=A^{\prime \prime}, \mathcal{M}=\mathcal{A}^{\prime \prime}, P=T$. Then we get a family $\mathcal{A}^{\prime}$ (parametrized by some connected but in general not irreducible curve). Now we use this family $\mathcal{A}^{\prime}$ and the sequence (4.1a) to apply 3.6 with $L=A^{\prime}, M=A$ and $\mathcal{L}=\mathcal{A}^{\prime}$. We get a new family $\mathcal{A}$. We denote by $T^{\prime}$ the curve parametrizing the family $\mathcal{A}$.

This family satisfies all the hypothesis of Lemma 1.1 (item (iv) is given by Proposition 3.3), and then Theorem I is proved.

\section{Moduli space of torsion-free sheaves on K3 surfaces.}

In this section we will apply Theorem I to prove Theorem II. The proof is similar to an argument in [G-H]. Recall that $S$ is a K3 complex surface. Given a line bundle $L$ on $S$ and an integer $c_{2}$, for any class $\zeta$ in the NeronSeveri group $N S(S)$ of $S$ satisfying $L^{2}-4 c_{2} \leq \zeta^{2}<0$ and $\zeta \equiv L \bmod 2$, we define the associated wall $W^{\zeta}=\{M: \zeta \cdot M=0$ and $\mathrm{M} \in$ ample cone $(\subset N S(S) \otimes \mathbb{R})\}$. We say that $W^{\zeta}$ is a wall of type $\left(L, c_{2}\right)$ (see [Q]). We say that a polarization is $\left(L, c_{2}\right)$-generic if it doesn't lie on any wall. In this case, semistability implies stability (i.e., there are no strictly semistable sheaves), the moduli space is smooth of dimension

$$
\operatorname{dim} \mathfrak{M}_{H}\left(L, c_{2}\right)=4 c_{2}-L^{2}-6,
$$

and then irreducibility is equivalent to connectivity (see [H-L2]).

First we prove that we can reduce the proof of Theorem II to a special case

Proposition 5.1. Assume that $\mathfrak{M}_{H}\left(L, c_{2}\right)$ is irreducible under the additional hypothesis that $\operatorname{Pic}(S)=\mathbb{Z}$. Then $\mathfrak{M}_{H}\left(L, c_{2}\right)$ is also irreducible under the conditions of Theorem II.

Proof. Let $S$ be a surface with an $\left(L, c_{2}\right)$-generic polarization $H$. By [G-H, 2.1.1], there is a connected family of surfaces $\mathcal{S}$ parametrized by a curve $T$ 
and a line bundle $\mathcal{L}$ on $\mathcal{S}$ such that $\left(\mathcal{S}_{0}, \mathcal{L}_{0}\right)=(S, L)$ and $\operatorname{Pic}\left(\mathcal{S}_{t}\right)=\mathcal{L}_{t} \cdot \mathbb{Z}$ for $t \neq 0$. By [G-H, prop. 2.2], there is a smooth proper family $\mathcal{Z} \rightarrow T$ such that $\mathcal{Z}_{0} \cong \mathfrak{M}_{H}\left(S, \mathcal{L}_{0}, c_{2}\right)$ (note that the polarization is $H$ and not $\mathcal{L}_{0}$ ) and $\mathcal{Z}_{t} \cong \mathfrak{M}_{\mathcal{L}_{t}}\left(\mathcal{S}_{t}, \mathcal{L}_{t}, c_{2}\right)$ for $t \neq 0$.

By hypothesis we know that $\mathcal{Z}_{t}$ is irreducible for $t \neq 0$, and then by $[\mathrm{H}$, III Ex 11.4], we obtain that $\mathcal{Z}_{0}$ is connected, but $\mathcal{Z}_{0}$ is smooth (because $H$ is generic), and then this implies that $\mathcal{Z}_{0}$ is irreducible.

Then from now on we will assume that $\operatorname{Pic}(S)=\mathbb{Z}$ and hence $H=L$ is the ample generator.

Proposition 5.2. Let $V$ be a torsion-free stable rank two sheaf with $c_{1}=L$, $c_{2} \leq \frac{1}{2} L^{2}+3$.

(a) Then $V$ fits in an exact sequence

$$
0 \rightarrow \mathcal{O}_{S} \rightarrow V \rightarrow L \otimes I_{Z} \rightarrow 0 .
$$

(b) Conversely, every nonsplit extension of $L \otimes I_{Z}$ by $\mathcal{O}_{S}$ is a torsion-free stable sheaf.

Proof. Take $V$ stable. Using the Riemann-Roch theorem,

$$
h^{0}(V)+h^{2}(V) \geq \frac{L^{2}}{2}-c_{2}+4 \geq 1 .
$$

If $h^{2}(V)$ were different from zero, by Serre duality we would have $\operatorname{Hom}(V, \mathcal{O}) \neq 0$, contradicting stability because this would give a nonzero morphism $V \rightarrow \mathcal{O}_{S}$.

Then $h^{0}(V) \neq 0$. Take a section of $V$. By stability, the quotient of the section is torsion-free, and we have an extension like (5.1). The extension is not split because $V$ is stable. This proves (a).

To prove part (b), assume $L^{\otimes m} \otimes I_{W}$ is a destabilizing subsheaf. Then $m>0$. By standard arguments we can assume that the quotient is torsionfree. The composition $L^{\otimes m} \otimes I_{W} \rightarrow V \rightarrow L \otimes I_{Z}$ is nonzero, because otherwise it would factor through $\mathcal{O}_{S}$ and this is impossible because $m>0$. Then $m=1$ and we have $I_{W} \hookrightarrow I_{Z}$. Furthermore, $l(W)>l(Z)$ because if $W=Z$, the sequence would split. Then we have a sequence

$$
0 \rightarrow L \otimes I_{W} \rightarrow V \rightarrow I_{W^{\prime}} \rightarrow 0
$$


but we reach a contradiction because $c_{2}=l(W)+l\left(W^{\prime}\right)>l(Z)+l\left(W^{\prime}\right)=$ $c_{2}+l\left(W^{\prime}\right)$. Then there is no destabilizing subsheaf, and $V$ is stable.

Consider the moduli space $\bar{M}$ of stable framed modules $(E, \alpha)$ where $E$ is a rank two torsion-free sheaf with $c_{1}(E)=L$ and second Chern class equal to $c_{2}$, and $\alpha: E \rightarrow L$ is a nontrivial homomorphism (see [H-L1]). The stability condition depends on a degree 1 polynomial $\delta(n)=\delta_{1} n+\delta_{0}$. If $0<\delta_{1}<L^{2}$, then $(E, \alpha)$ is stable iff $E$ is stable as a vector bundle (see [G-H, Lemma 1.1]). Let $N \subset \bar{M}$ be the subset corresponding to framed modules $(E, \alpha)$ such that $\operatorname{ker}(\alpha)=\mathcal{O}_{S}$. In [G-H, lemma 1.3], it is proved that $N$ is a closed subset. Note that $N$ can also be constructed as a moduli space of coherent systems [LP]. We have a diagram

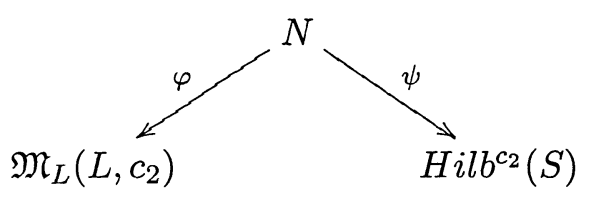

By Proposition 5.2(a) $\varphi$ is surjective, and by Proposition 5.2(b) the image of $\psi$ is

$$
X=\left\{Z \in \operatorname{Hilb}^{c_{2}}(S): \operatorname{dim} \operatorname{Ext}^{1}\left(L \otimes I_{Z}, \mathcal{O}_{S}\right) \geq 1\right\}
$$

Proposition 5.3. The set $X$ is connected.

Proof. By Serre duality and looking at the sequence

$$
0 \rightarrow H^{0}\left(L \otimes I_{Z}\right) \rightarrow H^{0}(L) \rightarrow H^{0}\left(\mathcal{O}_{Z}\right) \rightarrow H^{1}\left(L \otimes I_{Z}\right) \rightarrow 0
$$

we have $\operatorname{dim} \operatorname{Ext}^{1}\left(L \otimes I_{Z}, \mathcal{O}_{S}\right) \geq 1 \Longleftrightarrow h^{0}\left(L \otimes I_{Z}\right) \geq \frac{1}{2} L^{2}+3-c_{2}$. Now 
consider the following commutative diagram

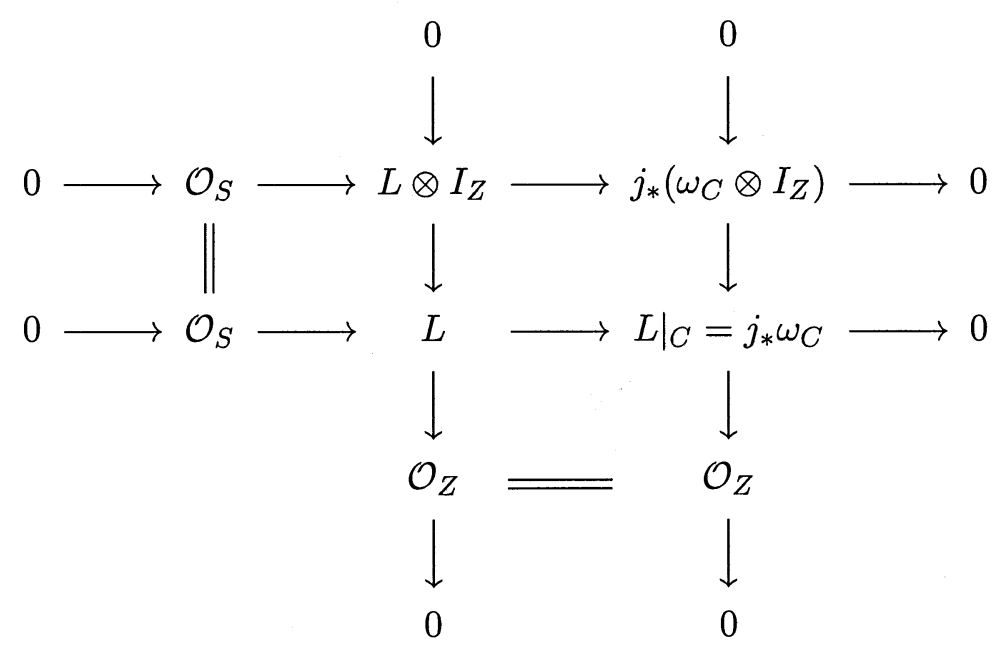

where $C \in \mathbb{P}\left(H^{0}\left(L \otimes I_{Z}\right)\right)$ (the curve $C$ might be singular, but we know it is irreducible and reduced because $\operatorname{Pic}(S)=\mathbb{Z}$ and $L$ primitive), $j: C \hookrightarrow S$ is the inclusion, and $\omega_{C}=\left.L\right|_{C}$ is the dualizing sheaf on $C$.

Using the top row we get $h^{0}\left(L \otimes I_{Z}\right) \geq \frac{1}{2} L^{2}+3-c_{2} \Longleftrightarrow h^{0}\left(\omega_{C} \otimes I_{Z}\right) \geq$ $\frac{1}{2} L^{2}+2-c_{2}$. This condition can be restated in terms of Brill-Noether sets $W_{d}^{r}$ :

$$
\omega_{C} \otimes I_{Z} \in W_{d}^{r}
$$

where $r=\frac{1}{2} L^{2}+1-c_{2}$, and $d=L^{2}-c_{2}$. We have

$$
\rho(r, d)=2 c_{2}-\frac{L^{2}}{2}-3=\frac{\operatorname{dim} \mathfrak{M}\left(L, c_{2}\right)}{2}>0,
$$

(recall that for $\operatorname{dim} \mathfrak{M}\left(L, c_{2}\right)=0$ the irreducibility of the moduli space is known by the work of Mukai [M]) and we can apply Theorem I. Now consider the variety

$$
N=\left\{(Z, C): Z \subset C, \operatorname{dim} \operatorname{Ext}^{1}\left(L \otimes I_{Z}, \mathcal{O}_{S}\right) \geq 1\right\} \subset \operatorname{Hilb}^{c_{2}}(S) \times \mathbb{P}\left(H^{0}(L)\right)
$$

and the projections

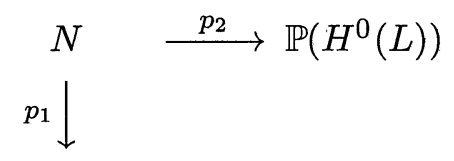

$\operatorname{Hilb}^{c_{2}}(S)$ 
By Theorem I, $p_{2}$ is surjective with connected fibers. Then $N$ is connected, and also the image of $p_{1}$, that is equal to $X$.

Finally we can prove Theorem II:

Proof. The fiber of $\psi$ (see diagram (5.2)) over a point corresponding to $Z$ is $\mathbb{P}\left(\operatorname{Ext}^{1}\left(L \otimes I_{Z}, \mathcal{O}_{S}\right)\right)$. In particular it is connected. By the previous proposition $X$ is connected, and then $N$ is also connected. Finally the surjectivity of $\varphi$ (Proposition 5.2(a)) proves that $\mathfrak{M}_{H}\left(H, c_{2}\right)$ is connected. This shows that the moduli space is irreducible under the additional hypothesis that $\operatorname{Pic}(S)=\mathbb{Z}$, and applying proposition 5.1 we obtain Theorem II.

Remark 2. Using similar techniques one can prove the irreducibility of the moduli space for any value of $c_{2}$. The proof is longer, due to the fact that we don't have a nice characterization of stable sheaves as in Proposition 5.2. The details are in [Go].

Acknowledgements. I would like to thank Robert Friedman, my thesis advisor, for introducing me to this problem, for encouragement and many discussions. This paper is part of my $\mathrm{PhD}$ thesis [Go]. I would also like to thank Ignacio Sols, for teaching me the fundamentals of algebraic geometry and for comments on a preliminary version of this paper (he suggested the present proof of lemma 1.1). I have also benefited from discussions with $\mathrm{R}$. MacPherson and R. Lazarsfeld.

This work was supported by the fellowship "Beca para ampliación de estudios en el extranjero" of the Banco de España.

\section{References.}

[AIK] A. Altman, A. Iarrobino and S. Kleiman, Irreducibility of the compactified Jacobian, Singularities of Real and Complex Maps (Proceedings of the Nordic Summer School, Oslo), 76, P. Holm Ed., (1977), 1-12.

[ACGH] E. Arbarello, M. Cornalba, P. Griffiths and J. Harris, Algebraic Curves, Grundlehren der mathematischen Wissenschaften, 267, Springer-Verlag, 1985. 
[D] C. D'Souza, Compactification of generalized Jacobians, Proc. Indian Acad. Sci. Sect. A Math. Sci., 88 (1979), 419-457.

[F-L] W. Fulton and R. Lazarsfeld, On the connectedness of degeneracy loci and special divisors, Acta Math., 146 (1981), 271-283.

[G-L] D. Gieseker and J. Li, Irreducibility of moduli of rank two vector bundles, J. Differ. Geom., 40 (1994), 23-104.

[Go] T. Gómez, Irreducibility of the moduli space of vector bundles on surfaces and Brill-Noether theory on singular curves, Princeton PhD Thesis, 1997; alg-geom/9710029.

[G-H] L. Göttsche and D. Huybrechts, Hodge numbers of moduli spaces of stable bundles on K3 surfaces, Internat. J. Math., 7 (1996), 359372 .

[H] R. Hartshorne, Algebraic Geometry, Grad. Texts in Math., 52, Springer Verlag, 1977.

[H-L1] D. Huybrechts and M. Lehn, Framed modules and their moduli, Internat. J. Math., 6 (1995), 297-324.

[H-L2] D. Huybrechts and M. Lehn, The geometry of moudli spaces of sheaves, Aspects of Mathematics, E31, Vieweg, BraunschweigWiesbaden, 1997.

[L] R. Lazarsfeld, Brill-Noether-Petri without degenerations, J. Differential Geometry, 23 (1986), 299-307.

[LP] J. Le Potier, Faisceaux semi-stables et systèmes cohérents, in 'Vector Bundles in Algebraic Geometry', Durham 93, Lond. Math. Soc. Lecture Notes Series, 208, 179-239.

[M] S. Mukai, On the moduli space of bundles on K3 surfaces I, in 'Vector bundles on algebraic varieties', Tata Institute of Fundamental Research studies in mathematics, Oxford University Press, 1987.

[O] G. Ottaviani, Varietá proiettive di codimensione piccola, Lecture notes INDAM, October 1994.

[O1] K. O'Grady, The irreducible components of moduli spaces of vector bundles on surfaces, Invent. Math., 112 (1993), 585-613. 
[O2] K. O'Grady, Moduli of vector bundles on projective surfaces: some basic results, Invent. Math., 123 (1994), 141-207.

[O3] K. O'Grady, The weight-two Hodge structure of moduli spaces of sheaves on a K3 surface, J. Algebraic Geom., 6 (1997) 599-644.

[Q] Z. Qin, Chamber structure of algebraic surfaces with Kodaira dimension zero and moduli spaces of stable rank two bundles, Math. Z., 207 (1991), 121-136.

[R] C.J. Rego, The compactified Jacobian, Ann. Scient. Ec. Norm. Sup. 4 sèrie, 13 (1980), 211-223.

[S] C. Simpson, Moduli of representations of the fundamental group of a smooth projective variety I, Publ. Math. I.H.E.S., 79 (1994), 47-129.

[Ye] Y.-G. Ye, Lagrangian subvarieties of the moduli space of stable vector bundles on a regular algebraic surface with $p_{g}>0$, Math. Ann., 295 (1993), 411-425.

[Yo] K. Yoshioka, An application of exceptional bundles to the moduli of stable sheaves on a K3 surface, alg-geom/9705027.

SCHOOL OF MATHEMATICS

Tata Institute of Fundamental Research

Homi BHabHa RoAd, Mumbai 400 005, India

E-mail address: tomas@math.tifr.res.in

ReCeIVed MaRCH 17, 1999. 